\title{
Imparting the Clinical Communication Skills to the Pharmacy Undergraduates
}

\author{
Leilei Ma \\ School of Chemistry \& Life Science \\ Weinan Normal University \\ Weinan, Shaanxi, China \\ e-mail: mary902@163.com
}

\author{
Yingjie Lei \\ School of Chemistry \& Chemical Engineering \\ Tianjin University of Technology \\ Tianjin, China \\ e-mail: yingjlei@163.com
}

\begin{abstract}
The academic agent on pharmacy has a vital role in the development of the licensed pharmacists. Aimed at the less informative imparting of clinical communication skills to the undergraduate students, the possible reforms, such as introducing the training materials, using the clinical cases and problem solving approaches, and setting up the effective assessment standard, are discussed, which will be benefit for the cultivation of the comprehensive communication skills so that the pharmaceutical care could be improved.
\end{abstract}

Keywords-Pharmacy undergraduate;

Clinical communication skills; Cultivation

\section{INTRODUCTION}

It is well-known that the quality of communication between patients and pharmacists is fundamental to providing effective pharmaceutical care. The concept of seven star pharmacists introduced by World Health Organization (WHO) has been adopted by International Pharmaceutical Federation (FIP) in 2000 in which a Communicator is one of the important sections. Therefore, the ability to communicate well with patients, such as understanding their concerns and beliefs, and eliciting the relevant information in order to make their personal decisions about drug treatment, should be an essential clinical skill. In most of the key schools of pharmacy in the developed countries, curriculum for communication skills are assessed in a variety of ways, including direct observation, use of simulated patients, and video recordings of interviews[1].1As for china, however, there is still not any such course for undergraduate curriculum to pharmacy and pharmaceutical engineering. In view of the global acceptance of the new role of a pharmacist as a pharmaceutical care provider, an extensive curriculum change and restructuring of education is urgently needed. Hence, we have established the teaching research and reform of pharmacy undergraduate curriculum several years ago. Herein, some experiences and advices on this issue from the actual situation of study existing are hoped to share.

This work was financially supported by the Educational Commission of Tianjin Municipality, China (No. C04-1007).

\section{Adopting THE RATIONAL TRAINING MATERIALS}

Early communication research has shown that communication skills play an important role in career advancement. Communication skills are crucial to helping pharmacy undergraduate develop their practice. Being a pharmacist will require one to communicate well orally and in writing with patients as well as presenting a professional image in writing skills. Meanwhile, better communication with pharmacists leads to a higher level of patient recall of information. It is clear that how to collect the rational training materials for the class becomes a focus for our teaching research.

In schools of pharmacy in the United Kingdom, communication skills are assessed in a variety of ways. Several validated patient rating scales have also been developed to assess the interpersonal skills of pharmacy students. Such methods of assessment are successful in measuring many aspects of students' interactions with patients. So the first thing we should do is to select some of the typical contents of communication skills from the relevant textbooks in English and adopt them to our training class.

There are many factors that can influence the process of communication. And there are many components to an interaction with another person. To have an interaction with another, one must be able to act as senders and receivers of messages. Therefore, skills including listening, responding, phrasing questions, and assertiveness, should be imparted to the students as a whole.

Take a part issue for listening and responding training as an example. We introduce a passage from the English textbook written by Ruth E. Nemire and Karen Kier[2].

Mr. Nodough is a patient who is picking up a prescription at the pharmacy. During the exchange, he says, "These prescription prices are so high. The pharmacist must be making a mint." Actually, many types of potential responses are possible.

(1) Judging_——"You shouldn't complain, Mr. Nodough. These medications are helping to keep you healthy."Suggests 
that someone should not have the feelings she does about a particular situation. The result is to invalidate the person's feelings and may discourage him from sharing feelings in the future.

(2) Reassuring ——_Don't worry about it; I'm sure you'll manage to figure out something." Tends to be given with good intentions to make someone feel better, but offers false reassurance since you do not know how things will work out. Often say it when people don't know what to say. The result is to invalidate the person's feelings.

(3) Probing - — "How much money do you spend on prescriptions each month?" Usually in the form of a question designed to elicit additional information. The result is it rather redirects the interaction taking focus away from the patient.

(4) Generalizing_—_Everyone feels that way sometimes." Or "I feel the same way when I get a prescription filled." Generalizes what the patient may be feeling. The result is some may feel that this type of response trivializes their feelings.

(5)Distracting — -Well, your prescription is ready now." Does not acknowledge what the patient has said; can be as drastic as a change of subject. The result is to invalidate the person's feelings, discourages patient from sharing feelings in the future.

(6) Advising - - "You should find a better health insurance company; one that covers prescriptions." Suggests what a person should do.

Offering alternatives is one way of helping the patient identify his own solution. Often, there is little you can do to solve their problem, but conveying that you have heard and understood it can go a long way

(7) Understanding - - "I understand you feel the prescription prices are too high." Allows you to convey your understanding of the message you received. This can be helpful, but you may not completely understand the patient's concern or situation. You will never be able to fully appreciate the patient's experience; to suggest you do may be offensive.

8)Empathy - "It must be very frustrating trying to pay for all your prescriptions and other costs of living." Goes beyond an understanding response to address the feelings that underlie a statement; demonstrates not only that you heard and understood what the person said, but also that you recognize the emotions that led him to say what he did. Acknowledges that you have heard the patient's concern and understand how it makes him feel.

Obviously, few types of potential responses will have the desired effect. And empathy is a very useful tool. By recognizing and responding to the feelings the person has, is a demonstration of true listening and also caring.

It is important to note that one of the key important elements of quality of care is the quality of interpersonal care and people presenting to a health professional expect good individual care. Therefore, how to set up the identified criteria that can be used to measure patients' perceptions of their interaction with pharmacy students is becoming increasingly recognized as important indicators. In accordance to this requirement, undergraduate pharmacy courses must provide clinical cases to practice.

\section{ENGAGING STUDENTS IN CLINICAL STITUTION}

Undergraduate students should have opportunities to participate in clinical research experiences and learn how to evaluate complex pharmaceutical problems from a variety of perspectives. A new method to train community pharmacists on counselling techniques for monitoring the use of nonprescription medicines was developed by the University of Sydney[3]. A much more successful approach to continuing professional education leading to adherence in the natural setting is ongoing training with immediate feedback and coaching through the use of pseudo customers. A pseudo customer, also known as a pseudo patron, pseudo patient, simulated patient, standardized patient, covert participant or mystery shopper, is an individual trained to go to a place, in this case a pharmacy, and present particular scenarios[4]. In the United Kingdom, the pseudo customer method was used to evaluate whether training in health promotion affected practice and to assess pharmacists' skills and knowledge in their response to symptoms[5].

Therefore, in our study we also used this practice to assess the effectiveness and efficiency of educational strategies to implement communication capabilities in response to symptoms in the community pharmacy setting. After documenting the counselling process, immediately after each visit, outside the pharmacy on an assessment form, the pseudo customer re-entered the pharmacy and gave detailed performance feedback to the pharmacist in charge in order to provide support for improving counselling skills and practice behaviour. From the result it can be seen that $95 \%$ of students could ask at least one question to check on accuracy of self-diagnosis in the cases of symptom presentation, even though different types of scenarios made a great difference to the spontaneity of questions.

\section{Setting UP EFFECTIVE ASSESSMENT}

In order to identify criteria for the assessment of communication skills in pharmacy students, we made an effort to invite two pharmacy experts from a local hospital to joint our team as co-counselors. Therefore, the need for such a tool is likely to stretch beyond the undergraduate curriculum. Pharmacy students must provide evidence that they are competent in communicating with patients as an essential requirement to practice as a pharmacist. Thus, the tool used in this study could be extremely useful in assessing and improving their communicate skills.

Development of the preliminary format of the questionnaire requires evaluation of a suitable response scale. We would like to use of a 5-point descriptor scale of excellent, very good, good, fair and poor at beginning, even though there are some discriminations with these criteria. Preparation of the final format of content structure and 
assessing the feasibility of using patients as assessors - is the basis of our ongoing work.

\section{LEARN TO CONDUCT A MEDICATION THERAPY INTERVIEW}

The interview is a goal-directed communication which is set for one person to obtain information from another. In order to get an effectiveness of communication with the patient, the pharmacy students should learn how to establish a medication therapy management interview.

Just remember try hard to satisfy the needs of the particular patient. It is better if the pharmacist has known something about the patients in advance, whether from previous visits or from discussions. What is more, it should be noted that the pharmacist's access to a patient's electronic medical record, such as essential data and laboratory results, may dramatically affect his or her ability to provide optimal direct patient-care services.

Be familiar with as much of the patient's information, such as social, medical, and medication information as possible. It is important to get to the point of the interview. Allow the patient to speak without interruptions. If necessary, try to use some verbal and nonverbal cues to keep the patient on track without significant interruption.

Ask a general question at the beginning of the interview, and then obtain the necessary demographic information. Remember to review the specific information obtained about the patient's medications, such as indication, dosing schedules, duration of therapy, and reasons for discontinuing.

Pay more attention to take special care when interacting with women to ensure issues such as medication effects in pregnancy and lactation are addressed.

\section{IMPART THE SKILLS OF MAKING THE PHARMACOTHERAPY Patient CASE Presentation}

During a case presentation, drug therapy problems are a good example. Case presentations are not like novels stories, so that student should learn how to tell the story of what found, what did, and what happened, on condition that you have already completed the assessment, identified drug therapy problems, constructed care plans, and may have evaluated your patient's outcomes.

Make a brief description of the patient. Practitioners need to see the patient to care for him or her. This should be simple, straightforward, and include how the patient appeared to be, which include age, gender, and physical description such as height, weight, and ethnic origin. Be sensitive to the words you use and how you say them.

Explain the main reason for the patient encounter. The description of the patient's reason for the encounter with the practitioner is the next step. The description should focus on the patient's initial request or the precipitating event, which might include items the patient does not understand, expressed concerns, or expectations that are unrealistic.
Using the patient's own words avoids adding the bias or interpretation onto the patient's description.

Write down the comprehensive medication history. A comprehensive description of the patient's medications involves several important areas, such as allergies, alerts, immunization records, and social drug use that may impact your decisions about medications as well as patient's current medical conditions at this time. The order to record medication history can be as follows, Indication-Drug Product-Dosage Regimen-Outcome. The summary of the assessment should include a brief review of clinical judgment regarding the patient, his/her active medical conditions, associated drug therapies, and any drug therapy problems identified. The summary should include the most important data that were used to make clinical decisions.

Identify the drug therapy problems. During the presentation of the patient's case, drug therapy problems must be stated clearly, if the drug therapy problems have been identified. There is a specific format used to describe a patient's drug therapy problems, firstly, the medical condition associated with the drug therapy problem. secondly, the drug therapy involved, and thirdly the relationship between the medical problem and the drug therapy.

After identifying the patient's drug-related needs, an organized care planning process should be followed. Therefore, the care plan should be organized and prioritized by active medical conditions and the problems should be presented in order of risk, severity, and importance to the patient.

Generally, a case presentation should be followed the above outline with respect to briefly describing the patient, the main conditions, and the drug therapies involved.

\section{LEARN TO BE A HEALTH EDUCATOR}

The pharmacy student is hoped to be an accessible health-care provider in a prime position to act as a health educator and a facilitator of patient empowerment. That is to say, students should master the fundamental knowledge so that to provide pertinent health-related information which enables the patient to make his or her own informed healthcare decisions. The key to educating patients is the act of engaging the patient and tailoring information to their lifestyle - not just telling them what to do.

For example, if you meet a patient with asthma who is severely allergic to pollen, you can tell him or her never go outside during pollen season. However, it is impractical and useless to the patient.

Instead, educate the patient to keep him or her mouth covered with the cup of hand or a small scarf in order to minimize the exposure to the pollen.

Engage the patient in adherence strategies is a good way to medication use is to by asking the patient what time he or she prefers to use controller inhaler and linking the 
medication use to daily routine, for instance, before breakfast and at bedtime. Recommend any pertinent changes in technique to make sure that the patient learn how to adopt it.

\section{CONCLUSION}

According to the actual teaching situation, the teaching reform of pharmacists' communication curriculum is researched. The contents of communication skills teaching as well as assessment are proposed. It is recognized by students, internal colleagues and external experts.

\section{REFERENCES}

[1] James D, Nastasic S, Horne R. "The design and evaluation of a simulated-patient teaching programme to develop the consultation skills of undergraduate pharmacy students," Pharm World Sci, 2001,23: 212-216

[2] Ruth E. Nemire, Karen Kier. 2009. Pharmacy Student Survival Guide, Second Edition (ed): New York, The McGraw- Hill Companies, Inc, pp. 51-57.

[3] Neto A. "Changing pharmacy practice: the Australian experience". The Pharmaceutical Journal, 2003, 270, 235-236

[4] Neto A, Kelly F, Benrimoj SI. "Shaping practice behavior: novel training methodology". The International Journal of Pharmacy Practice, 2001, 9, 203-210

[5] Anderson C. "A controlled study of the effect of a health promotion training scheme on pharmacists' advice about smoking cessation". Journal of Social and Administrative Pharmacy, 1995, 12,115-124 